\title{
PARTICIPACIÓN SINDICAL Y SALUD LABORAL: UNA RELACIÓN POSITIVA
}

\section{LABOR UNION PARTICIPATION AND OCCUPATIONAL HEALTH: A POSITIVE RELATIONSHIP}

\author{
Raúl Payá Castiblanque \\ Pere J. Beneyto Calatayud \\ Universidad de Valencia. Valencia/España \\ raul.paya@uv.es \\ pere.j.beneyto@uv.es
}

Recibido/Received: 29/05/2018

Modificado/Modified: 23/07/2018

Aceptado/Accepted: 10/09/2018

\section{RESUMEN}

Después de casi diez años de reducción progresiva de los registros estadísticos de accidentes de trabajo, la confluencia entre crisis económica y reforma laboral ha provocado, desde 2013, un repunte de la siniestralidad, paralelo al incremento de la precariedad contractual, la contracción de las políticas públicas y empresariales en materia de salud laboral, así como de la reducción de la cobertura y funciones de la representatividad sindical. El artículo revisa la regulación normativa y evolución reciente del modelo español de prevención de riesgos laborales, tanto físicos como psico-sociales, indagando sobre las causas y efectos de la correlación detectada entre sus principales indicadores (de prevención, formación, siniestralidad y evaluación) con los del sistema de participación de los trabajadores, tanto genérica (delegados sindicales y comités de empresa) como especializada (delegados de prevención y comités de salud y seguridad), concluyendo con diversas propuestas orientadas a reforzar y ampliar dicha intervención sindical.

\section{PALABRAS CLAVE}

Crisis; precariedad; riesgos laborales; intervención sindical.

\section{SUMARIO}

1. Introducción. 2. Crisis económica, reforma laboral y empleo precario. 3. Participación de los trabajadores en la prevención de riesgos laborales. 3.1. Representación (general y específica) de los trabajadores en la empresa. 3.2 Correlación entre la representación de los trabajadores y la prevención de riesgos. 4. Conclusiones. Bibliografía.

\footnotetext{
ABSTRACT

After more than a decade of progressive decline in statistical records of accidents at work, the confluence between economic crisis and labor reform has led, since 2013, a rebound in the loss ratio, parallel to the increase in contractual precariousness, the contraction of public and business policies in the field of occupational health, as well as the reduction of coverage and functions of union representation. The article reviews the normative regulation and recent evolution of the Spanish model of prevention of occupational risks, both physical and psycho-social, investigating the causes and
} 
effects of the correlation detected among its main indicators (prevention, training, accident rate and evaluation) with those of the employee participation system, both generic (union delegates and works councils) and specialized (prevention delegates and health and safety committees), concluding with various proposals aimed at strengthening and expanding this union intervention.

\title{
KEYWORDS
}

Crisis; Precariousness; Labor Risks; Union Intervention.

\section{CONTENTS}

1. Introduction. 2. Economic crisis, labor reform and precarious employment. 3. Participation of workers in the prevention of occupational risks. 3.1. Representation (general and specific) of the workers in the company. 3.2 Correlation between the representation of workers and the prevention of risks. 4. Conclusions. References.

\author{
A Elena \\ Una entre miles de jóvenes víctimas de accidentes de trabajo \\ Para que recupere pronto la mano y la alegría.
}

\section{INTRODUCCIÓN}

Con la aprobación en 1989 de la Directiva-Marco 89/391-CEE (Boletín Oficial del Estado $\mathrm{n}^{\circ} 183$ ), la Unión Europea inauguraba una nueva etapa en materia de prevención de riesgos en el trabajo y promoción de la salud laboral. La posterior ratificación de dicha Directiva por los estados miembros (con más de cinco años de retraso en el caso español) y la aplicación creciente de sus disposiciones en las empresas, tendría un importante impacto en la reducción de la siniestralidad, identificándose como un factor decisivo en dicho proceso la participación de los trabajadores y sus representantes en tanto que tienen derecho a solicitar al empresario que tome las medidas adecuadas y a presentarle propuestas para paliar cualquier riesgo para los trabajadores y/o eliminar las fuentes de riesgo laboral (art. 11.3 de la Directiva Marco).

Mientras que los índices de incidencia de la siniestralidad laboral (accidentes de trabajo con baja laboral por cada 100.000 trabajadores) presentan en los principales países europeos una reducción constante y sostenida desde hace décadas, independientemente del ciclo económico, su evolución en el caso español parece mucho más sensible a las oscilaciones del mismo. Según se desprende de la correspondiente serie histórica (Benavides et al, 2008), en 1984 se registró un punto de inflexión y posterior alza del índice de siniestralidad tras quince años de una caída sostenida, manteniéndose desde entonces una tendencia ascendente hasta los primeros años 2000 , cuando se invierte de nuevo, pero entonces a la baja, coincidiendo con la etapa de crecimiento económico más largo de nuestra historia reciente y la progresiva aplicación de las medidas establecidas por la Ley 31/1995 de Prevención de Riesgos Laborales (en adelante LPRL) (BOE $\left.\mathrm{n}^{\circ} 269\right)$.

La crisis iniciada en 2008 supondrá primero una ralentización en la reducción de dicho índice, durante la fase recesiva del ciclo, y su posterior aumento a partir de 2013 coincidiendo con una recuperación económica tan frágil como desigual (gráfica 1), en una secuencia que parece avalar la hipótesis planteada por algunos investigadores (Terrés et al. 2004) según la cual el aumento de los accidentes laborales constituiría una especie de "peaje" o "daño colateral" del crecimiento económico. 
Especialmente significativa resulta la desigual distribución intersectorial del índice de siniestralidad que en el caso de la construcción dobla ampliamente la media, mientras que en los servicios se reduce a la mitad de los niveles registrados en la industria.

Gráfica 1: Índice de siniestralidad laboral en España (2004-2016), por sectores económicos

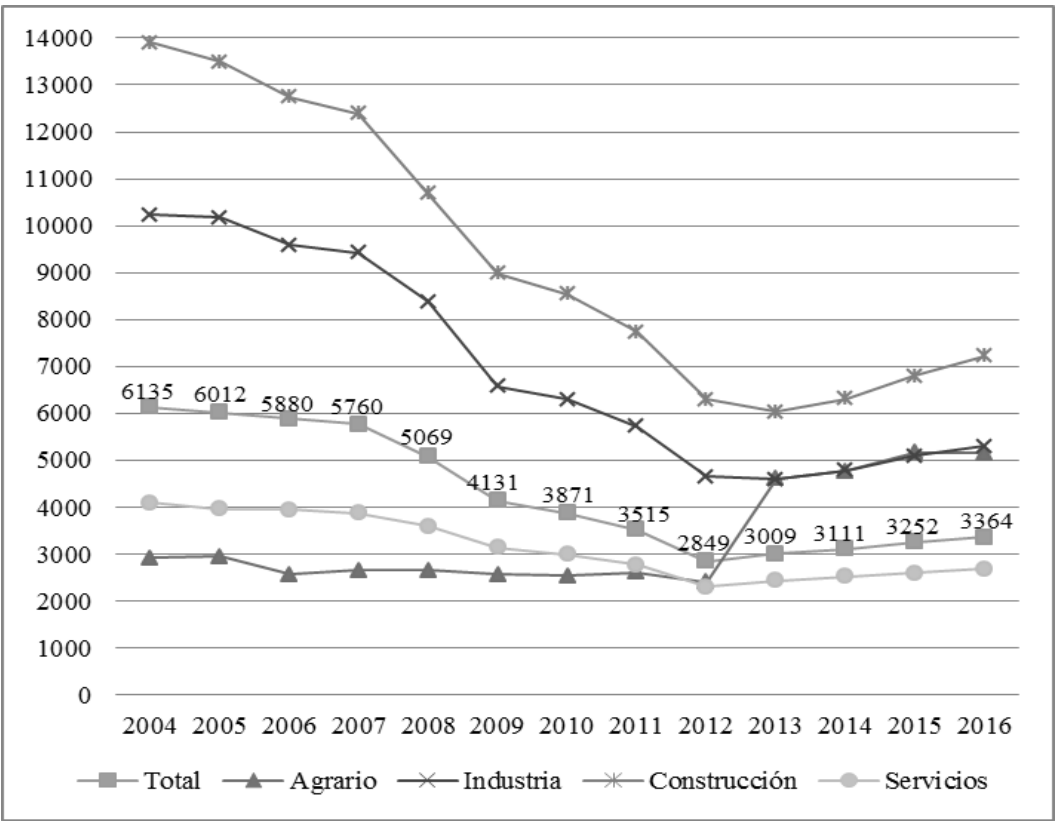

Fuente: Anuario de Estadísticas Laborales. Condiciones de Trabajo y Relaciones Laborales. Accidentes de trabajo, (Ministerio de Trabajo, 2004-2017). Elaboración propia

Los datos oficiales sitúan en 2012 el punto de inflexión de la siniestralidad laboral cuya tasa de incidencia experimentaría desde entonces y hasta finales de 2017 un incremento del 17,1\% (tabla 1), describiendo una tendencia radicalmente opuesta a la de la Unión Europea cuya tasa es prácticamente la mitad de la española y ha seguido reduciéndose durante estos años. La reforma laboral de 2012 constituye un factor clave en la inversión de dicha tendencia pues impuso una agresiva desregulación del mercado de trabajo y de las relaciones laborales, con el consiguiente aumento de la precariedad contractual, debilitamiento de las instituciones de participación de los trabajadores y reducción de la cobertura y eficacia de la negociación colectiva (Alós, et al. 2017), procesos todos ellos que numerosos estudios nacionales (Boix y Vogel, 2006; García et al. 2015; Linares y López, 2016) e internacionales (Lewchuk et al., 2003; Walters y Nichols, 2007; Fulton, 2013) han demostrado que ejercen una influencia decisiva sobre la evolución de la siniestralidad laboral.

\section{CRISIS ECONÓMICA, REFORMA LABORAL Y EMPLEO PRECARIO}

Para contextualizar adecuadamente el impacto de la crisis, la contrarreforma laboral así como las dificultades para la participación sindical, tanto general como específica, en materia 
de prevención de riesgos, conviene analizar, siquiera sea esquemáticamente, la evolución reciente y situación actual de los principales indicadores socioeconómicos.

Tabla 1: Evolución del índice de siniestralidad laboral en España y la Unión Europea, 2007-2017

\begin{tabular}{|l|l|l|}
\hline Año & España & Unión Europea \\
\hline 2007 & 5.760 & 2.736 \\
\hline 2008 & 5.069 & n.d. \\
\hline 2009 & 4.131 & 1.843 \\
\hline 2010 & 3.871 & 1.961 \\
\hline 2011 & 3.515 & 1.886 \\
\hline 2012 & 2.848 & 1.723 \\
\hline 2013 & 3.009 & 1.701 \\
\hline 2014 & 3.111 & 1.647 \\
\hline 2015 & 3.252 & 1.569 \\
\hline 2016 & 3.364 & 1.521 \\
\hline $2017^{*}$ & 3.334 & n.d. \\
\hline
\end{tabular}

Fuente: European Statistical Office (Eurostat, 2018). (*) Datos provisionales. Elaboración propia

El estallido en 2008 de una crisis económica de alcance global puso de manifiesto la vulnerabilidad del modelo español de crecimiento basado, en gran parte, en sectores de bajo valor añadido, orientado por la demanda de bienes inmuebles y servicios, intensivos en empleos precarios y no cualificados, favorecido por bajas tasas de interés, etc. lo que explica el impacto diferencial respecto de otras economías de nuestro entorno, fundamentalmente en materia de empleo, atendiendo tanto a su evolución cuantitativa como a su composición cualitativa.

Tabla 2: Expedientes de regulación de empleo (2007-2017)

\begin{tabular}{|l|l|l|l|l|l|}
\hline Año & Empresas & \multicolumn{5}{|l|}{ Trabajadores afectados } \\
\cline { 3 - 6 } & & $\begin{array}{l}\text { Despidos } \\
\text { colectivos }\end{array}$ & $\begin{array}{l}\text { Suspensión } \\
\text { contrato }\end{array}$ & $\begin{array}{l}\text { Reducción } \\
\text { jornada }\end{array}$ & TOTAL \\
\hline 2007 & 4.020 & 25.742 & 32.433 & 226 & 58.401 \\
\hline 2008 & 6.249 & 40.572 & 104.841 & 2.675 & 148.088 \\
\hline 2009 & 19.434 & 63.476 & 465.215 & 20.591 & 549.282 \\
\hline 2010 & 17.269 & 52.534 & 211.942 & 38.270 & 302.746 \\
\hline 2011 & 21.168 & 67.981 & 215.012 & 60.636 & 343.629 \\
\hline 2012 & 27.570 & 82.876 & 300.713 & 99.724 & 483.313 \\
\hline 2013 & 21.228 & 70.351 & 234.116 & 75.505 & 379.972 \\
\hline 2014 & 10.637 & 35.875 & 92.234 & 31.457 & 159.566 \\
\hline 2015 & 5.675 & 24.572 & 62.298 & 13.652 & 100.522 \\
\hline 2016 & 3.999 & 24.348 & 53.658 & 8.570 & 86.576 \\
\hline 2017 & 2.615 & 20.813 & 28.257 & 8.427 & 57.497 \\
\hline Total & 150.133 & 509.140 & 1.800 .719 & 359.733 & 2.669 .592 \\
\hline
\end{tabular}

Fuente: Boletín de Estadísticas Laborales (Ministerio de Trabajo, 2007-2017). Elaboración propia. 
Lo que caracteriza al modelo español es el uso excesivo que la norma laboral permite hacer de la contratación temporal, el elevado fraude en la contratación y la progresiva especialización de la economía en actividades y segmentos de negocio en los que la fuerza de trabajo es fácilmente sustituible, lo que retroalimenta la elevada temporalidad y rotación laboral.

Entre 2007 y 2017 se registraron un total de 150.133 expedientes de regulación de empleo que afectaron a 2.669.592 trabajadores (equivalente al 15,9\% de la población asalariada actual), de los que más medio millón fueron despedidos (tabla 2), poniendo de manifiesto las facilidades flexibilizadoras derivadas de la desregulación laboral (eliminación de la autorización administrativa para los despidos colectivos y de la acreditación de pérdidas para justificar la extinción, siendo suficiente la simple reducción de ventas durante tres trimestres consecutivos).

La precariedad contractual sería, pues, la variable de ajuste dominante frente a la recesión económica, tanto en términos de rescisión y ajuste de plantillas como de modalidades de contratación. Así vemos cómo en los años más duros de la crisis (2007-2013) se destruyeron casi tres millones de empleos (tabla 3), iniciándose desde entonces una lenta y desigual recuperación que sitúa el número de trabajadores asalariados a finales de 2017 en 15.792.200, aún inferior en casi siete puntos porcentuales al registrado antes de la crisis.

Tabla 3: Evolución de la contratación y temporalidad laboral (2007-2017)

\begin{tabular}{|l|l|l|l|l|c|c|}
\hline \multirow{2}{*}{ Año } & \multicolumn{3}{|l|}{ Contratos registrados } & \multicolumn{3}{|c|}{ Asalariados } \\
\cline { 2 - 7 } & Total & Temporales & $\%$ & Total & Temporales & $\%$ \\
\hline 2007 & $18.621,1$ & $16.401,7$ & 88,1 & $17.094,6$ & $5.442,2$ & 31,8 \\
\hline 2008 & $16.601,2$ & $14.698,6$ & 88,5 & $16.920,1$ & $4.965,5$ & 29,3 \\
\hline 2009 & $14.021,8$ & $12.709,4$ & 90,6 & $15.803,8$ & $4.073,4$ & 25,8 \\
\hline 2010 & $14.417,2$ & $13.188,9$ & 91,5 & $15.705,1$ & $3.981,1$ & 25,3 \\
\hline 2011 & $14.433,3$ & $13.323,1$ & 92,3 & $15.479,3$ & $3.814,2$ & 24,6 \\
\hline 2012 & $14.421,0$ & $12.808,0$ & 89,9 & $14.560,9$ & $3.474,1$ & 23,8 \\
\hline 2013 & $13.792,6$ & $13.657,7$ & 92,3 & $14.123,9$ & $3.397,3$ & 24,1 \\
\hline 2014 & $16.727,1$ & $15.376,8$ & 91,9 & $14.413,1$ & $3.552,1$ & 24,6 \\
\hline 2015 & $18.576,3$ & $17.067,1$ & 91,8 & $14.948,9$ & $3.909,7$ & 26,1 \\
\hline 2016 & $19.979,0$ & $18.265,5$ & 91,4 & $15.404,6$ & $4.152,3$ & 26,9 \\
\hline 2017 & $21.501,3$ & $19.572,0$ & 91,0 & $15.792,2$ & $4.252,2$ & 26,6 \\
\hline
\end{tabular}

Fuente.- Boletín de Estadísticas Laborales (Ministerio de Trabajo, 2007-2017) y Encuesta de Población Activa (EPA) (Instituto Nacional de Estadística, 2018). Elaboración propia.

Entre finales de 2013 y el mismo período de 2017 se crearon 1.668 .300 empleos asalariados, de los que más de la mitad eran temporales, habiendo repuntado la tasa correspondiente del 24,1 al 26,6 por cien (la segunda más alta de la UE, después de Polonia), poniendo de manifiesto la escasa calidad del empleo generado durante la recuperación, con contratos de duración cada vez menor (50,6 días de media en 2017), lo que dificulta notablemente la consolidación de pautas preventivas en materia de seguridad laboral.

En este sentido, resulta especialmente significativo el impacto de la crisis y la reforma laboral sobre la salud y la seguridad de los asalariados, el $37 \%$ de los cuales declara estar expuesto a riesgos en el desarrollo de su actividad, según la última Encuesta Nacional de Condiciones de Trabajo (Instituto Nacional de Seguridad e Higiene en el Trabajo, 2017), habiendo aumentado dicho porcentaje en diez puntos durante los últimos años, en paralelo a 
los cambios en las condiciones de empleo, el incremento de la precariedad contractual y la contracción de las políticas públicas y empresariales en materia de salud laboral (formación, cambio organizativo, inspección), quebrando con ello la línea descendente inaugurada por la LPRL.

Por su parte, la Encuesta Nacional de Gestión de Riesgos Laborales en las empresas española (INSHT, 2014), realizada en centros de más de cinco trabajadores, distingue entre los tradicionales riesgos físicos (ergonómicos y derivados del manejo de máquinas y herramientas, que afectarían entre el $25 \mathrm{y}$ el 80 por cien de las empresas) y los riesgos nuevos y emergentes de tipo psicosocial (estrés, inseguridad en el empleo, horario irregular, discriminación..., con niveles de exposición entre el 10 y el 50 por cien).

Asimismo, el 47\% de los trabajadores habrían experimentado cambios importantes en sus condiciones de empleo en el último año (incremento de las horas de trabajo y de las tareas a desarrollar por una parte y, por otra, reducción de su salario), al tiempo que el $21 \%$ de los centros de trabajo se han visto afectados por reestructuraciones laborales.

La modalidad contractual deviene, en definitiva, en factor determinante de la siniestralidad laboral (tabla 4) de forma que entre los trabajadores temporales la tasa de incidencia dobla prácticamente la de los fijos que apenas registra un ligero cambio de tendencia en su evolución sostenida a la baja, mientras que la de los contratados temporales estaría repuntando fuertemente desde 2012.

La segmentación del mercado de trabajo derivada de la crisis (altas tasas de paro y precarización contractual), junto con los efectos desreguladores de la reforma a los que ya hemos hecho referencia, han agravado la asimetría de las relaciones laborales y erosionado los recursos e instituciones de participación de los trabajadores (sindicatos, representación en la empresa, negociación colectiva, diálogo social), debilitando objetivamente su capacidad de hacer frente a los efectos más devastadores de la recesión, entre los que se incluye el deterioro de las condiciones de trabajo y consiguiente incremento de los riesgos laborales tanto físicos como psico-sociales.

Tabla 4: Accidentes con baja laboral, según tipo de contrato (2007-2017) Índice de siniestralidad (IS)

\begin{tabular}{|l|l|l|l|l|l|}
\hline \multirow{2}{*}{ Año } & Total & \multicolumn{2}{|c|}{ Contrato Indefinido } & \multicolumn{2}{c|}{ Contrato Temporal } \\
\cline { 2 - 6 } & Núm. & Núm. & IS & Núm. & IS \\
\hline 2007 & 924.981 & 459.254 & 3.942 & 454.834 & 8.360 \\
\hline 2008 & 804.959 & 439.310 & 3.676 & 351.309 & 7.083 \\
\hline 2009 & 617.440 & 378.839 & 3.229 & 226.402 & 5.563 \\
\hline 2010 & 569.523 & 355.384 & 3.032 & 201.536 & 5.063 \\
\hline 2011 & 512.584 & 321.879 & 2.760 & 175.953 & 4.618 \\
\hline 2012 & 408.537 & 266.209 & 2.402 & 129.834 & 3.742 \\
\hline 2013 & 404.284 & 265.019 & 2.470 & 127.216 & 3.753 \\
\hline 2014 & 424.625 & 268.117 & 2.469 & 144.548 & 4.072 \\
\hline 2015 & 458.023 & 277.394 & 2.513 & 169.430 & 4.333 \\
\hline 2016 & 489.065 & 288.324 & 2.563 & 189.750 & 4.572 \\
\hline 2017 & 503.749 & --- & --- & --- & -- \\
\hline
\end{tabular}

Fuente: Anuario de Estadísticas Laborales. Condiciones de Trabajo y Relaciones Laborales. Accidentes de trabajo, (Ministerio de Trabajo, 2004-2017). Elaboración propia

En cuanto a la negociación colectiva, ámbito en el que se gestionan medidas y planes de 
salud laboral y prevención de riesgos en tanto que la LPRL es una norma de derecho mínimo indispensable que puede ser mejorada y desarrollada por convenio colectivo (art. 2.2 de la LPRL), el impacto de la crisis y la reforma laboral ha sido desigual (tabla 5). En un primer momento, las facilidades para el descuelgue y la imposición de fecha de caducidad del contenido normativo de los convenios provocó un importante freno en el ritmo de negociación, con una reducción en los niveles de cobertura de empresas y trabajadores que se iría estabilizando a partir de mayo de 2013, por la firma del Acuerdo sobre ultra-actividad (comisión de seguimiento del II AENC), hasta situarse actualmente en tono a un $15 \%$ por debajo de los niveles registrados al inicio de la crisis.

Tabla 5: Convenios, empresas y trabajadores (2007-2017) Según ámbito funcional, año de efectos económicos y período de registro

\begin{tabular}{|l|c|l|l|l|l|l|}
\hline & \multicolumn{2}{|c|}{ Total } & \multicolumn{2}{c|}{ De empresa } & \multicolumn{2}{c|}{ De ámbito superior } \\
\hline Año & Conv. & Trabajadores & Conv. & Trabajadores & Conv. & Trabajadores \\
\hline 2007 & 6.016 & 11.606 .500 & 4.598 & 1.261 .000 & 1.418 & 10.345 .400 \\
\hline 2008 & 5.987 & 11.968 .100 & 4.539 & 1.215 .300 & 1.448 & 10.752 .900 \\
\hline 2009 & 5.689 & 11.557 .800 & 4.323 & 1.114 .600 & 1.366 & 10.443 .200 \\
\hline 2010 & 5.067 & 10.794 .300 & 3.802 & 923.200 & 1.265 & 9.871 .100 \\
\hline 2011 & 4.585 & 10.662 .800 & 3.422 & 929.000 & 1.163 & 9733.800 \\
\hline 2012 & 4.376 & 10.099 .000 & 3.234 & 925.700 & 1.142 & 9.173 .300 \\
\hline 2013 & 4.589 & 10.265 .400 & 3.395 & 932.700 & 1.194 & 9.332 .700 \\
\hline 2014 & 5.185 & 10.304 .700 & 4.004 & 867.200 & 1.181 & 9.437 .500 \\
\hline 2015 & 5.642 & 10.227 .300 & 4.493 & 846.900 & 1.149 & 9.380 .400 \\
\hline $2016^{*}$ & 4.886 & 9.608 .100 & 3.559 & 661.700 & 970 & 8.946 .400 \\
\hline $2017^{*}$ & 3.249 & 7.069 .400 & 2.460 & 479.500 & 789 & 6.589 .900 \\
\hline
\end{tabular}

Fuente: Estadística de Convenios Colectivos de Trabajo (ECCT) (Ministerio de Trabajo, 2007-2017) (*) datos provisionales. Elaboración propia

En cuanto a los contenidos de la negociación colectiva, las cláusulas relativas a la prevención de riesgos laborales han experimentado una significativa reducción en los últimos años, según los datos del Ministerio de Empelo. Así, el establecimiento de Planes de prevención habría descendido desde 2010 en que figuraba en el 49,3\% de los convenios y afectaba al 31,6\% de los trabajadores, hasta 2015 (último año del que se dispone de datos consolidados) en el que su cobertura se limitaba al $22,8 \%$ de los convenios y el $15,9 \%$ de los trabajadores.

Por su parte, la posibilidad de ampliar el número de delegados de prevención, sobre los legalmente establecidos por la LPRL, habría pasado en el mismo período de estar regulada por el $11,9 \%$ de los convenios para el $23,8 \%$ de los trabajadores al 8,3 y 12,6 por cien, respectivamente.

Así pues, la convergencia entre crisis económica y reforma laboral habría afectado significativamente a la negociación colectiva, tanto en su dimensión cuantitativa (reducción de cobertura) como cualitativa (restricciones de contenido), con el consiguiente impacto sobre los programas y actores de la prevención de riesgos laborales.

Cabe añadir, finalmente, que las políticas de recortes y austeridad impuestas por el gobierno conservador han contribuido también a debilitar los sistemas de inspección ya que, según el último Informe anual de la Inspección de Trabajo y Seguridad Social (Ministerio de Empleo y Seguridad Social, 2016), se habría reducido el número de inspectores (de 1.878 en 
2013 a 1.792 en 2016) y, en consecuencia, restado eficacia a su actividad, ya de por sí difícil habida cuenta de la elevada ratio de cobertura (10.800 trabajadores aproximadamente por inspector).

Se trata, además, de un sistema de inspección generalista, diferente al de los países escandinavos, anglosajones y germánicos que disponen de un sistema de inspección especializado en salud laboral (Castejón y Crespán, 2007), lo que en nuestro caso supone la acumulación de funciones, por cuanto los inspectores nacionales deben vigilar, controlar y sancionar a las empresas infractoras tanto en las cuestiones referidas a la prevención de riesgos laborales como a cualquier otra del orden social. De hecho, el citado Informe pone de manifiesto que solo el 28\% de las actuaciones realizadas en 2016 fueron efectuadas para tratar aspectos preventivos frente al $53 \%$ en materia de cotizaciones a la seguridad social, lo que explicaría el débil efecto de la Inspección de Trabajo sobre los indicadores de accidentabilidad laboral.

\section{PARTICIPACIÓN DE LOS TRABAJADORES EN LA PREVENCIÓN DE RIESGOS LABORALES}

Además de los factores contextuales (ciclo económico, evolución del empleo) y de las modalidades contractuales (sector de actividad y tamaño de empresa, etc.) que condicionan la evolución de la siniestralidad laboral, la regulación normativa y los dispositivos preventivos y de participación de los trabajadores desempeñan, en nuestra opinión, un papel fundamental no suficientemente ponderado, por lo que pretendemos centrarnos ahora en su análisis y evaluación.

La LPRL 31/1995 define un modelo de gestión preventiva y participativa, en cumplimiento tanto de un mandato constitucional (art. 40.2 de la CE) como de la correspondiente directiva europea. Especial atención merece a estos efectos el capítulo $\mathrm{V}$ de la LPRL sobre "Consulta y participación de los trabajadores y trabajadoras", cuyo artículo 33 considera obligatoria la consulta previa a la planificación y organización del trabajo y la introducción de nuevas tecnologías. Por su parte, el artículo 34 establece la elección, de entre los representantes de los trabajadores, de Delegados de Prevención (DP) en empresas de 6 y más empleados, y el artículo 38 define la composición (paritaria y colegiada) y las funciones (de información, consulta y participación propositiva) de los Comités de Seguridad y Salud (CSS). Se trata, pues, de una representación específica para las funciones de salud laboral y prevención de riesgos, con carácter complementario, cuando no compartido e incluso solapado (Linares y López, 2016), con la representación general de los trabajadores (delegados de personal y comités de empresa), regulada por los artículos 62 y 63 del correspondiente Estatuto.

Es por ello que, antes de proceder al análisis de correlación entre participación de los trabajadores y gestión preventiva de riesgos laborales, consideramos necesario revisar la información disponible sobre la estructura y evolución reciente de ambas modalidades de representación.

\subsection{Representación (general y específica) de los trabajadores en la empresa}

La representación de los trabajadores en las empresas se articula tanto a través de la afiliación directa en las secciones sindicales (SSE) como de diversos mecanismos electorales (delegados, comités de empresa, de salud laboral), actuando en ambos casos como un factor determinante en la mejora de las relaciones laborales (Beneyto, 2014) 
La última Encuesta Europea sobre las Condiciones de Trabajo (EWCS) (European Foundation for the Improvement of Living and Working Conditions, 2015) informa que prácticamente la mitad de los trabajadores de la UE-28 disponen en su empresa de representación sindical (SSE-Comité), si bien dicha tasa media varía considerablemente (gráfica 2), alcanzando niveles de cobertura próximos al 80\% en los países escandinavos y de apenas un tercio en los del área oriental, situándose los países latinos y centroeuropeos en torno a la media.

Gráfica 2: Representación de los trabajadores en la empresa

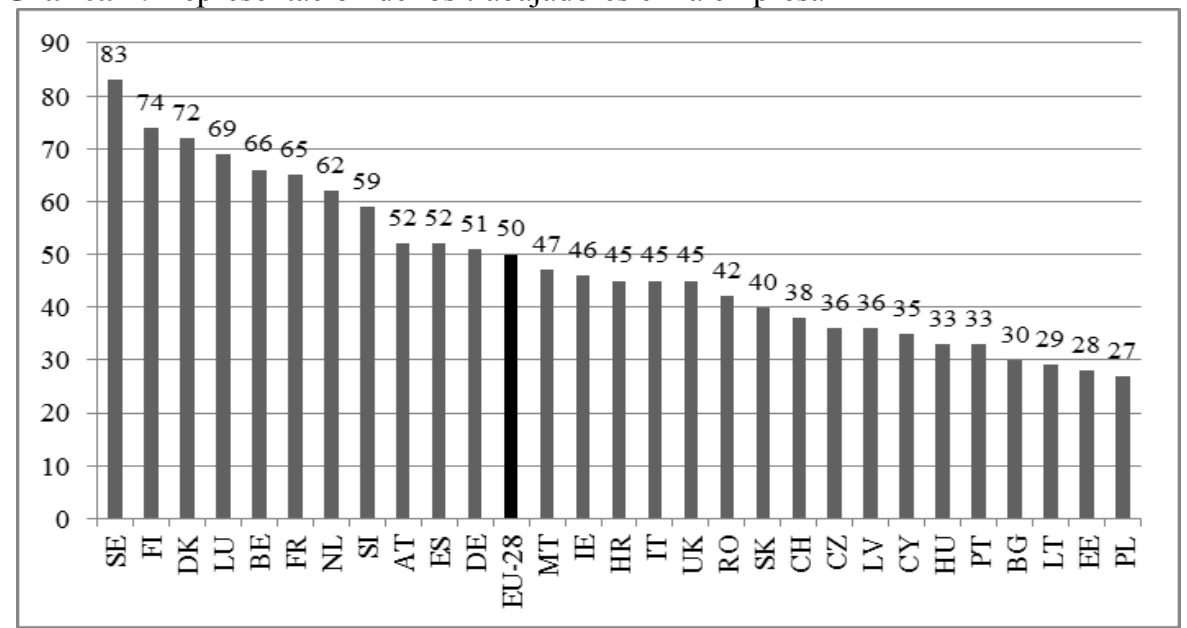

Fuente: Sixth European Working Conditions Survey (EWCS) (Eurofound, 2015). Elaboración propia

Gráfica 3: Cobertura sindical, según sector y tamaño de las empresas

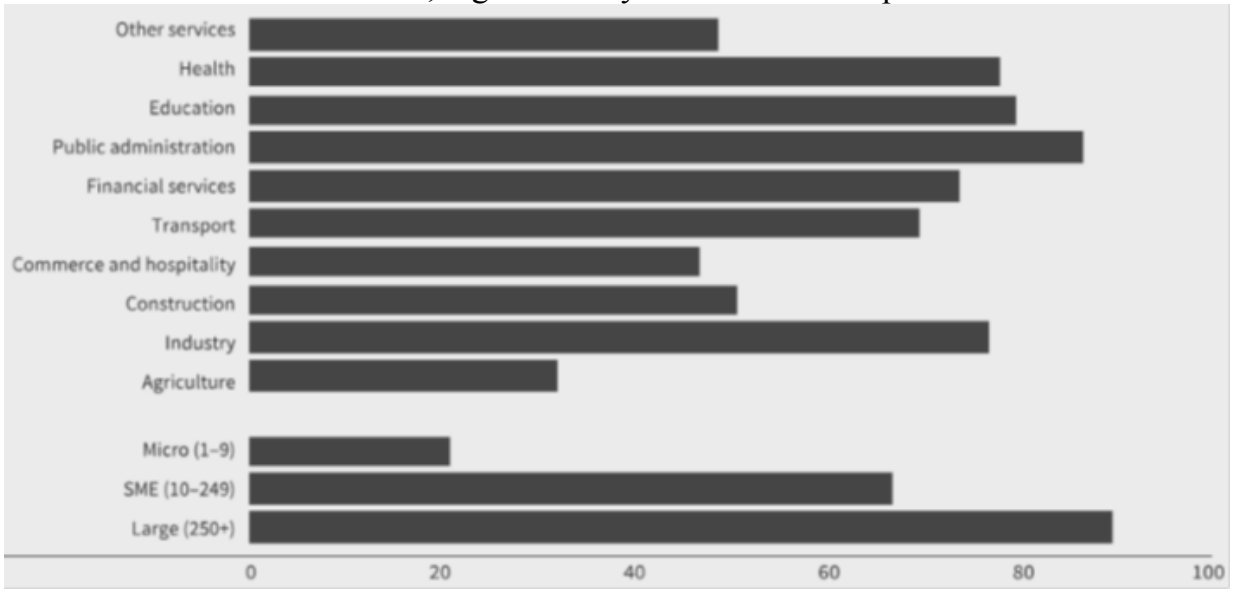

Fuente: Sixth European Working Conditions Survey (EWCS) (Eurofound, 2015)

La cobertura de la representación sindical presenta, asimismo, pautas diferenciadas por sectores de actividad y tamaño de las empresas (gráfica 3), siendo considerablemente mayor 
en las grandes empresas, los servicios públicos y la industria manufacturera que en las microempresas, la construcción y los servicios privados.

Esta vía de representación sindical se completa con la dedicada a funciones de prevención de riesgos y promoción de la salud laboral en los centros de trabajo (gráfica 4), que habrían alcanzado ya una tasa de cobertura media del 58\% de los trabajadores europeos y aproximadamente del $50 \%$ en el caso español.

Ambas formas de representación mantienen entre sí relaciones diversas según los países, desde vías paralelas a superpuestas y/o complementarias, con una cobertura agregada en torno al $58 \%$ del total de trabajadores.

Gráfica 4: Cobertura de los delegados y comités de salud laboral

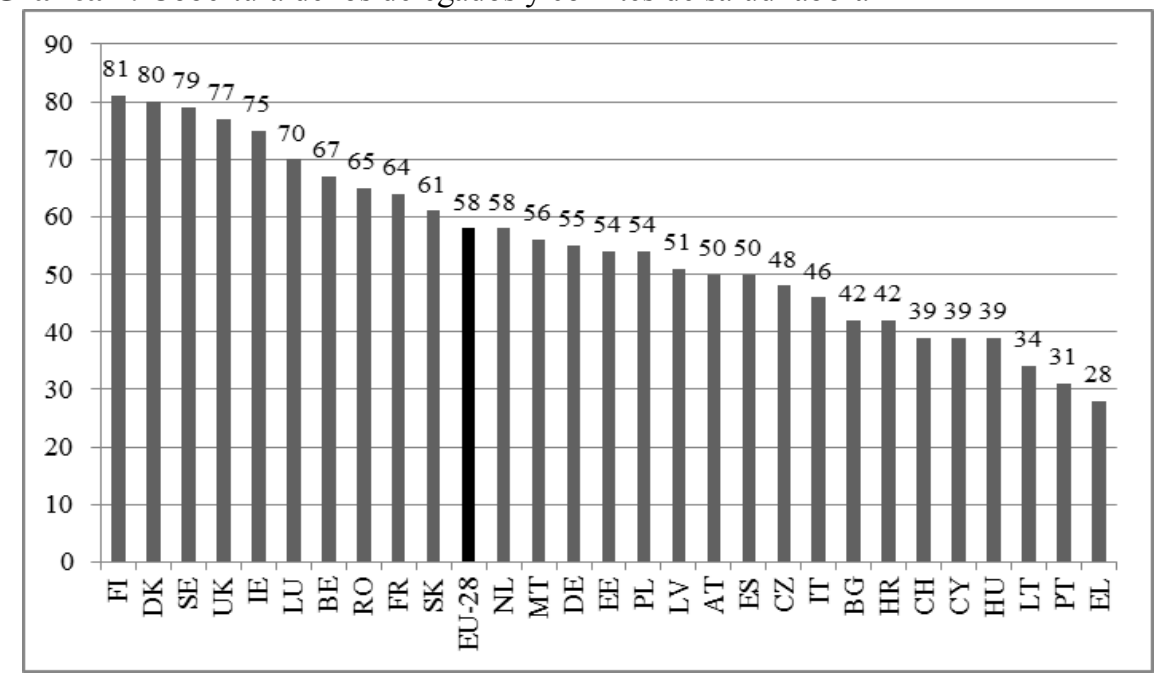

Fuente: Sixth European Working Conditions Survey (EWCS) (Eurofound, 2015). Elaboración propia

Por su parte, el modelo dual de representación vigente en nuestro país introduce mecanismos de expansión de la cobertura sindical que partiendo de unas tasas de afiliación y presencia relativamente bajas (entre el 16 y el 19 por cien de la población asalariada), logra triplicar su representatividad mediante mecanismos de audiencia electoral y cuadriplicar incluso su influencia a través de su participación en la negociación colectiva.

El Estatuto de los Trabajadores regula el derecho de representación colectiva en base a la elección de delegados de personal y comités de empresa, correspondiendo los primeros a los centros de trabajo de entre 10 y 49 empleados y los segundos a los de más de 50.

Por su parte, la LPRL establece que los órganos de representación especializada en salud laboral y prevención de riesgos serán elegidos por y entre los representantes del personal (art. 35.2), confiriendo a la negociación colectiva la posibilidad de promover otros mecanismos de designación (art. 35.4).

Dicho mecanismo de configuración de la representación específica en materia de prevención de riesgos genera procesos ambivalentes pues si, por una parte, garantiza la expansión de su cobertura, por otra provoca solapamientos y acumulación de tareas representativas en las mismas personas, especialmente en las empresas más pequeñas, que disponen de un solo crédito horario para el desarrollo de sus dos funciones. 
En cuanto a la representación sindical de ámbito general, los datos oficiales permiten constatar que desde la primera convocatoria (1978) la evolución de su estructura y cobertura y resultados (tabla 6), refleja tanto las oscilaciones del cambio económico y ocupacional, como la recomposición interna del movimiento sindical, en períodos que siguen inicialmente los avatares políticos y, después, los ciclos económicos.

Las cuatro primeras convocatorias de elecciones sindicales se celebraron sólo en empresas del sector privado, mientras que a partir de 1987 el sistema se amplió, con algunas peculiaridades formales (distinta definición de las unidades electorales y distribución de las Juntas de Personal), a los funcionarios de la Administración Pública, Sanidad, Enseñanza, Justicia, etc., tras la aprobación de la Ley 9/1987 de Órganos de Representación, Determinación de las condiciones de trabajo y Participación del personal al servicio de las Administraciones Pública $\left(\mathrm{BOE}, \mathrm{n}^{\mathrm{0}} 144\right)$, lo que aumentó notablemente su cobertura y representatividad.

Tabla 6: Elecciones sindicales en España (1978-2018)

\begin{tabular}{|l|l|l|l|l|r|l|l|l|r|}
\hline Año & Total & \multicolumn{2}{|c|}{ CC.OO. } & \multicolumn{2}{c|}{ UGT } & \multicolumn{2}{c|}{ Otros } & \multicolumn{2}{c|}{ No afiliados } \\
\cline { 3 - 10 } & delegados & Núm. & $\%$ & Núm. & $\%$ & Núm. & $\%$ & Núm. & $\%$ \\
\hline 1978 & 193.112 & 66.540 & 34,5 & 41.897 & 21,7 & 25.953 & 13,4 & 58.725 & 30,4 \\
\hline 1980 & 164.617 & 50.817 & 30,8 & 48.194 & 29,3 & 22.053 & 13,4 & 43.553 & 26,5 \\
\hline 1982 & 140.770 & 47.016 & 33,4 & 51.672 & 36,7 & 25.058 & 17,8 & 17.024 & 12,1 \\
\hline 1986 & 175.363 & 59.230 & 33,8 & 69.427 & 39,6 & 33.998 & 19,4 & 12.708 & 7,2 \\
\hline 1990 & 237.261 & 87.730 & 36,9 & 99.737 & 42,0 & 41.387 & 17,4 & 8.407 & 3,5 \\
\hline 1995 & 204.586 & 77.348 & 37,8 & 71.112 & 34,7 & 49.495 & 24,2 & 6.631 & 3,2 \\
\hline 1999 & 260.285 & 98.440 & 37,8 & 96.770 & 37,2 & 57.006 & 21,9 & 8.969 & 3,1 \\
\hline 2003 & 283.075 & 110.208 & 38,9 & 103.805 & 36,7 & 69.062 & 24,4 & -- & -- \\
\hline 2007 & 312.017 & 122.079 & 39,1 & 114.973 & 36,8 & 74.965 & 24,0 & -- & -- \\
\hline 2012 & 305.200 & 114.505 & 37,5 & 107.983 & 35,4 & 76.834 & 25,2 & 5.878 & 1,9 \\
\hline 2016 & 266.103 & 95.423 & 35,8 & 86.344 & 32,4 & 78.753 & 29,6 & 5.583 & 2,1 \\
\hline 2018 & 271.725 & 96.773 & 35,6 & 88.306 & 32,5 & 86.646 & 31,9 & -- & - \\
\hline
\end{tabular}

Fuente: Ministerios de Trabajo y AAPP para los datos de 1978 a 1990; SIGIS-CCOO, para las restantes convocatorias

Se trata, pues, de un sistema de representación caracterizado por un alto grado de consolidación (12 elecciones en 40 años), cuyas principales fortalezas son su expansión sostenida hasta 2007, concentración en torno a CC.OO. y UGT que acumulan más de dos tercios del total, pero que presenta, también, debilidades importantes, especialmente las derivadas de su desigual nivel de cobertura y reciente evolución a la baja.

En el primer caso, la Encuesta de Calidad de Vida en el Trabajo (ECVT) (Ministerio de Trabajo, 2010) (tabla 7), realizada por el Ministerio de Trabajo desde 1999 e interrumpida en 2010, sitúa la cobertura directa del sistema de representación sindical en el 47,4\% sobre el total de la población asalariada (alrededor de 6’5 millones de trabajadores). Se trata, con todo, de valores mínimos (en el 15,3\% de los casos no consta una respuesta clara al respecto) y desigualmente repartidos según tamaño de empresa y sector de actividad.

Por su parte, la crisis económica, con su correlato de cierre de empresas, reducción de 
plantillas, etc., habría provocado una reducción del 14,7\% en el número de representantes sindicales elegidos entre 2007 y 2016, que en los últimos años registraría una significativa recuperación.

Tabla 7: Cobertura de la representación sindical (I) (\% de trabajadores que declaran tener representación en su empresa)

\begin{tabular}{|c|c|c|c|}
\hline & Sí & No & $\mathrm{Ns} / \mathrm{Nc}$ \\
\hline TOTAL & 47,4 & 37,2 & 15,3 \\
\hline \multicolumn{4}{|c|}{ SEGÚN TAMAÑO DE LA EMPRESA } \\
\hline De 1 a 9 trabajadores & 25,2 & 57,2 & 17,6 \\
\hline De 10 a 49 & 48,8 & 35,6 & 15,6 \\
\hline De 50 a 249 & 66,9 & 17,9 & 15,2 \\
\hline De 250 y más trabajadores & 81,9 & 9,6 & 8,5 \\
\hline \multicolumn{4}{|c|}{ SEGÚN SECTOR DE ACTIVIDAD } \\
\hline Agricultura & 31,6 & 43,5 & 24,9 \\
\hline Industria & 57,0 & 31,6 & 11,4 \\
\hline Construcción & 31,5 & 52,8 & 15,7 \\
\hline Comercio y Hostelería & 34,3 & 47,7 & 18,0 \\
\hline Transportes y comunicaciones & 59,7 & 31,6 & 8,7 \\
\hline Servicios financieros & 64,3 & 24,8 & 10,9 \\
\hline Servicios empresariales & 40,0 & 41,9 & 18,1 \\
\hline Servicios públicos & 61,5 & 23,9 & 14,6 \\
\hline Servicios personales y a la comunidad & 17,5 & 62,2 & 20,2 \\
\hline
\end{tabular}

Fuente: Encuesta de Calidad de Vida en el Trabajo (ECVT) (Ministerio de Empleo, 2010)

Por su parte, un estudio sobre los cuatro últimos períodos electorales, promovido por la Fundación $1^{\circ}$ de Mayo (Alós, et al. 2015), pone de manifiesto los límites del sistema de representación sindical, derivados de la atomización de la estructura empresarial española (tabla 8), que excluye del mismo a tres de cada cuatro empresas y a uno de cada seis trabajadores, al establecer que sólo podrán convocarse elecciones a delegados o comités en centros de trabajo del sector privado con una plantilla mínima de 6 empleados (art. 62.1 del Estatuto de los Trabajadores).

El número total de empresas susceptibles de realizar elecciones aumentó en un 15,5\% en los años de expansión analizados (2003-2007) para reducirse en un 17,1\% con la crisis, siendo 293.000 en la actualidad. Sin embargo, son más de un millón las micro-empresas que quedan excluidas del sistema electoral.

Tabla 8: Empresas y trabajadores inscritos en la Seguridad Social, según tamaño de la plantilla, 2017

\begin{tabular}{|c|l|l|l|l|}
\hline & \multicolumn{2}{|l|}{ Empresas } & \multicolumn{2}{l|}{ Trabajadores } \\
\cline { 2 - 5 } & Núm. & $\%$ & Núm. & $\%$ \\
\hline De 1 a 5 trabajadores & 1.005 .256 & 77,4 & 2.026 .119 & 16,2 \\
\hline De 6 a 9 & 124.848 & 9,6 & 897.378 & 7,1 \\
\hline De 10 a 49 & 141.931 & 10,9 & 2.750 .199 & 21,9 \\
\hline De 50 a 249 & 22.224 & 1,7 & 2.165 .254 & 17,3 \\
\hline De 250 y más & 4.053 & 0,3 & 4.701 .306 & 37,5 \\
\hline TOTAL & 1.298 .312 & 100 & 12.540 .256 & 100 \\
\hline
\end{tabular}

Fuente: Anuario de Estadísticas Laborales. Afiliación de Trabajadores al Sistema de la Seguridad Social (Ministerio de Trabajo, 2017). Elaboración propia 
Como ya se ha indicado, la representación sindical genérica se complementa con la de carácter específico dedicada a funciones de prevención de riesgos y promoción de la salud laboral conforme a lo establecido en el artículo 35 de la LPRL.

Según la Encuesta Nacional de Gestión de Riesgos Laborales en las Empresas Española (INSHT, 2014) (gráfica 5), la red de delegados de prevención alcanza una cobertura media del $49,9 \%$ de los centros de trabajo con más de 5 trabajadores, variando sustancialmente según el tamaño de la plantilla (del $41,1 \%$ en las empresas de entre 6 y 9 trabajadores y el $94,8 \%$ en las de más de 250 ) y el sector de actividad (del $46,2 \%$ en comercio y hostelería al $60,2 \%$ en la industria manufacturera).

Gráfica 5: Niveles de cobertura de la representación (general y específica) de los trabajadores en la empresa

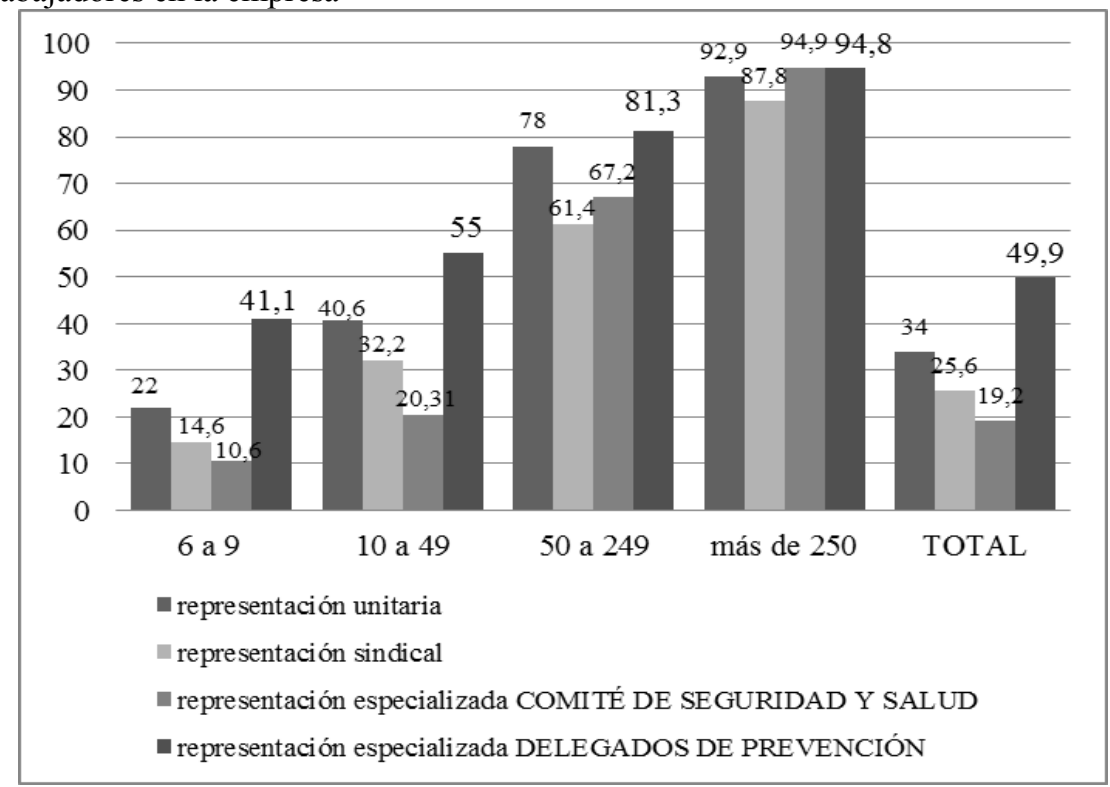

Fuente: Encuesta ESENER-2-España (INSHT, 2014). Elaboración propia

Esta red de delegados de salud laboral en las empresas estaría integrada, en el momento de su máxima expansión anterior a la crisis, por unos 187.000 representantes (Narocki et al. 2011), desempeñado un importante papel en el desarrollo de la nueva cultura de la prevención y contribuido decisivamente a la reducción de la siniestralidad entre 2000 y 2012 (del 7.558,4 al 2.848,9), experimentando desde entonces un preocupante repunte provocado fundamentalmente, como ya se ha explicado, por el aumento de la precariedad y desregulación generados de la reforma laboral, a lo que habría que añadir el efecto derivado de la reciente reducción de la representación sindical, como acreditan los últimos estudios cualitativos de la ESENER-2 (European Agency for Safety and Health at Work, 2017; Santos et al. 2017) .

Con todo, la tasa de cobertura en nuestro país de los representantes especializados en prevención de riesgos es notablemente inferior a la de los principales países europeos (gráfica 6), destacando los altos niveles registrados en el sistema de relaciones laborales escandinavo, seguido del centroeuropeo y anglosajón y, en el área latina, el caso italiano explicable por la 
existencia de "delegados territoriales" de salud laboral que cubren transversalmente las empresas, especialmente PYMEs, que no disponen de representación propia (Fulton, 2013).

Gráfica 6: Cobertura de la representatividad en salud laboral en Europa

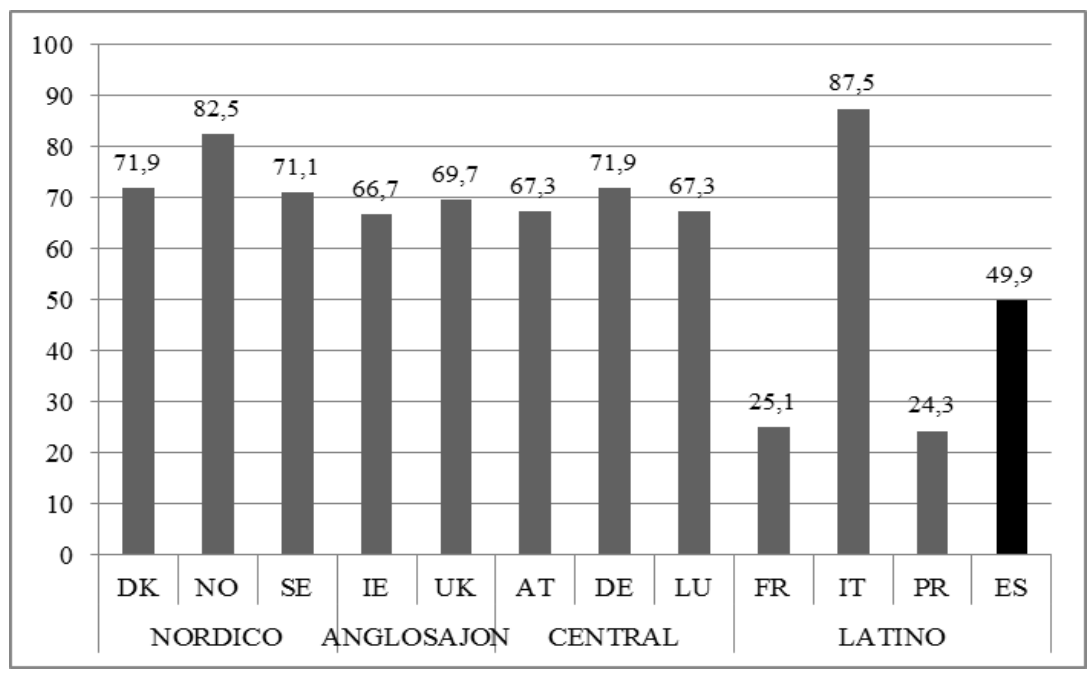

Fuente: Encuesta ESENER-2 (EU-OSHA, 2014). Elaboración propia

Esta experiencia podría aplicarse también en el caso español, desarrollando a tal efecto lo previsto en el artículo 35.4 de la LPRL que determina que los convenios colectivos podrán establecer nuevos sistemas de designación de delegados de prevención siempre que la representación unitaria o los trabajadores ejerzan el derecho de designación (Payá, 2014).

\subsection{Correlación entre la representación de los trabajadores y la prevención de riesgos}

Una vez analizada la estructura y cobertura de los órganos de representación (general y específica) de los trabajadores en las empresas, nos centraremos ahora en desarrollar la hipótesis central de nuestra investigación y evaluar el impacto de la participación de dichos representantes en la identificación, prevención y reducción de riesgos laborales.

A tal efecto, compararemos mediante modelos de regresión lineal los datos de representatividad según el tamaño de empresa registrados en la Encuesta Nacional de Gestión de Riesgos Laborales en las empresas españolas (INSHT, 2014), parte integrante de la encuesta europea ESENER-2 (EU-OSHA, 2014) (vid. Gráfica 5), con los indicadores sobre las diferentes fases del proceso de evaluación de riesgos, empezando por la identificación de los factores organizacionales que pueden ser disfuncionales o de riesgos, para posteriormente analizar la planificación de acciones de prevención y controlar dichos factores de riesgo reduciendo su impacto para, finalmente, identificar los planes y programas para su prevención y control.

Según la ESENER-2 (EU-OSHA, 2014) la evaluación de riesgos laborales en las empresas de España y la UE alcanza niveles similares (gráfica 7) siendo, en ambos casos, mayor la evaluación preventiva de riesgos físicos que la de carácter psicosocial: mientras que los primeros presentan tasas de evaluación superiores al $80 \%$, la de los segundos no supera el $60 \%$. 
Por su parte, la Encuesta Nacional de Gestión de la Seguridad y Salud en las Empresas (ENGE) (INSHT, 2009) acredita que las actividades preventivas (tabla 9) son mucho más frecuentes en los centros de trabajo que cuentan don delegados de personal (DP) que en los que carecen de dicha representación.

Gráfica 7: Evaluación de riesgos por especialidad preventiva, en España y a UE

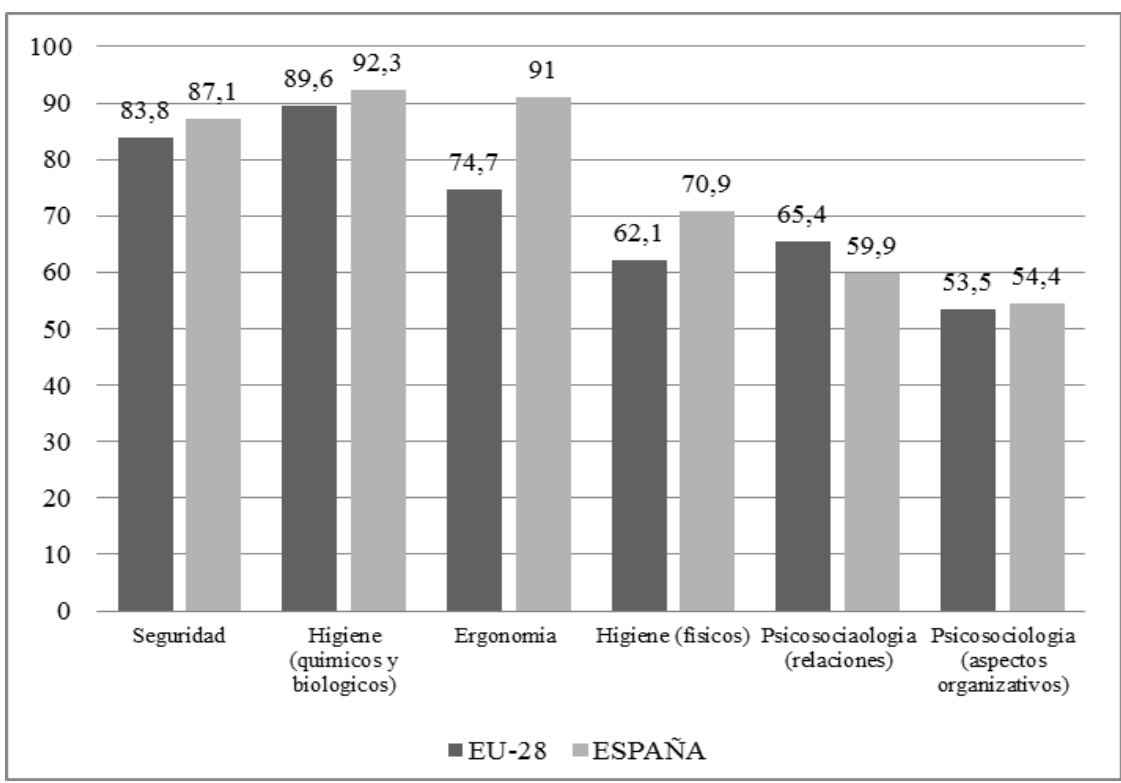

Fuente: Encuesta ESENER-2 (EU-OSHA, 2014). Elaboración propia

Tabla 9: Actividades preventivas realizadas según la existencia o no de delegados de prevención

\begin{tabular}{|l|c|c|}
\hline $\begin{array}{l}\text { Actividades preventivas } \\
\text { Datos en \% }\end{array}$ & $\begin{array}{c}\text { Centros de trabajo } \\
\text { CON DP }\end{array}$ & $\begin{array}{c}\text { Centros de trabajo } \\
\text { SIN DP }\end{array}$ \\
\hline Reconocimientos médicos en el último año & 92,8 & 74,0 \\
\hline Evaluación de riesgos & 93,5 & 70,4 \\
\hline Elaboración del Plan de Prevención & 82,9 & 54,8 \\
\hline Información de RL y de las medidas adoptadas & 73,5 & 51,8 \\
\hline Formación en materia de salud laboral & 70,0 & 50,6 \\
\hline Planificación actividad preventiva & 77,2 & 47,0 \\
\hline Definición de las medidas de emergencia & 63,3 & 45,2 \\
\hline Establecimiento de prioridades y controles & 60,6 & 36,3 \\
\hline $\begin{array}{l}\text { Obligación de incluir la prevención en todas las } \\
\text { actividades }\end{array}$ & 50,5 & 27,4 \\
\hline Investigación de accidentes de trabajo & 47,3 & 26,3 \\
\hline Elaboración de un Plan de autoprotección & 44,5 & 24,1 \\
\hline Prácticas derivadas del Plan de emergencia & 42,5 & 20,4 \\
\hline
\end{tabular}

Fuente: Encuesta ENGE (INSHT, 2009)

En este punto, nuestra investigación (gráfica 8) constata la existencia de una fuerte 
correlación positiva entre la existencia de representantes y la realización de actividades preventivas ( $\mathrm{r} 2=0,94 ; \mathrm{p}<0,07$ seguridad; $\mathrm{r}^{2}=0,99 ; \mathrm{p}<0,04 \mathrm{y} \mathrm{r}^{2}=0,99 ; \mathrm{p}<0,2$ higiene industrial; $r^{2}=0,97 ; p<0,04$ ergonomía; $r^{2}=0,88 ; p<0,01$ y $r=0.95 ; p<0,02$ psicosociología, relaciones y condiciones, respectivamente).

Gráfica 8: Correlación entre el nivel de representatividad y el desarrollo de actividades de evaluación de riesgos

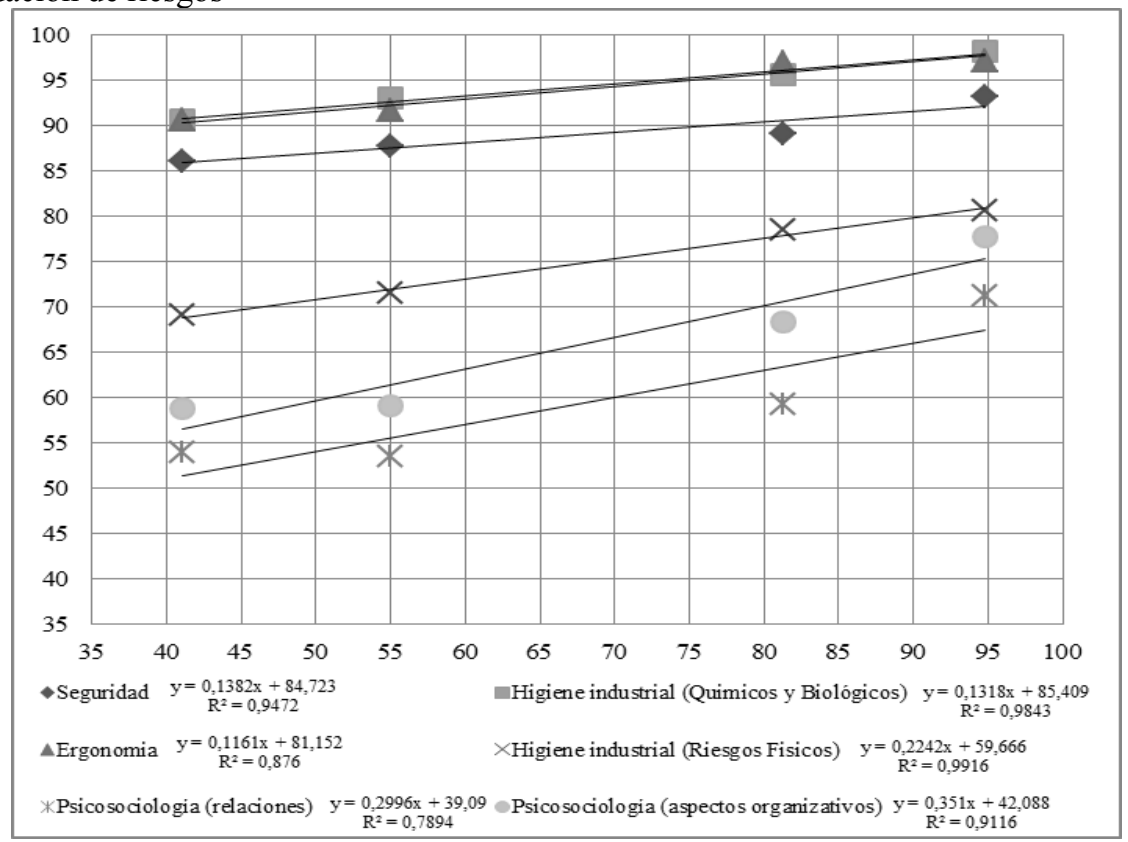

Fuente: Encuestas ESENER-2-España (INSHT, 2014) y ESENER-2 (EU-OSHA, 2014). Elaboración propia

El impacto de la participación de los representantes de los trabajadores en la evaluación de riesgos es sensiblemente mayor en el caso de los de carácter psicosocial, sobre todo en empresas de más de 50 trabajadores donde existe un comité de seguridad y salud laboral mostrando mayor impacto en la evaluación de condiciones de trabajo (horarios, estilos de dirección, vacaciones, espacio físico de trabajo, sistema de comunicación / información, sistema de evaluación / remuneración, etc.). En este sentido, la presencia de delegados de prevención en los centros de trabajo opera como factor explicativo del 78,9\% y $91,1 \%$ de evaluaciones de riesgos que contemplan los riesgos de origen psicosocial ( $2=0,789$ relaciones y $\mathrm{r} 2=0,911$ condiciones de trabajo).

Una vez identificado su impacto positivo sobre la evaluación de riesgos psicosociales en los centros de trabajo, el análisis se centra en identificar el nivel de participación de los representantes en las siguientes fases del proceso, es decir, la planificación de medidas preventivas (gráficas 9 y 10) y, si se supera el riesgo, el establecimiento de planes y procedimientos preventivos (gráfica 11).

En referencia a la planificación de medidas preventivas la encuesta ESENER-2 identifica, para el caso de los riesgos psicosociales, las siguientes medidas de actuación:

1) Reorganización del trabajo con el fin de reducir las exigencias y presión laboral. 
2) Asesoramiento confidencial para trabajadores.

3) Puesta en marca de procedimientos de resolución de conflictos.

4) Intervención en caso de demasiadas horas de trabajo o un horario irregular.

Gráfica 9: Correlación entre el nivel de representatividad y la planificación preventiva

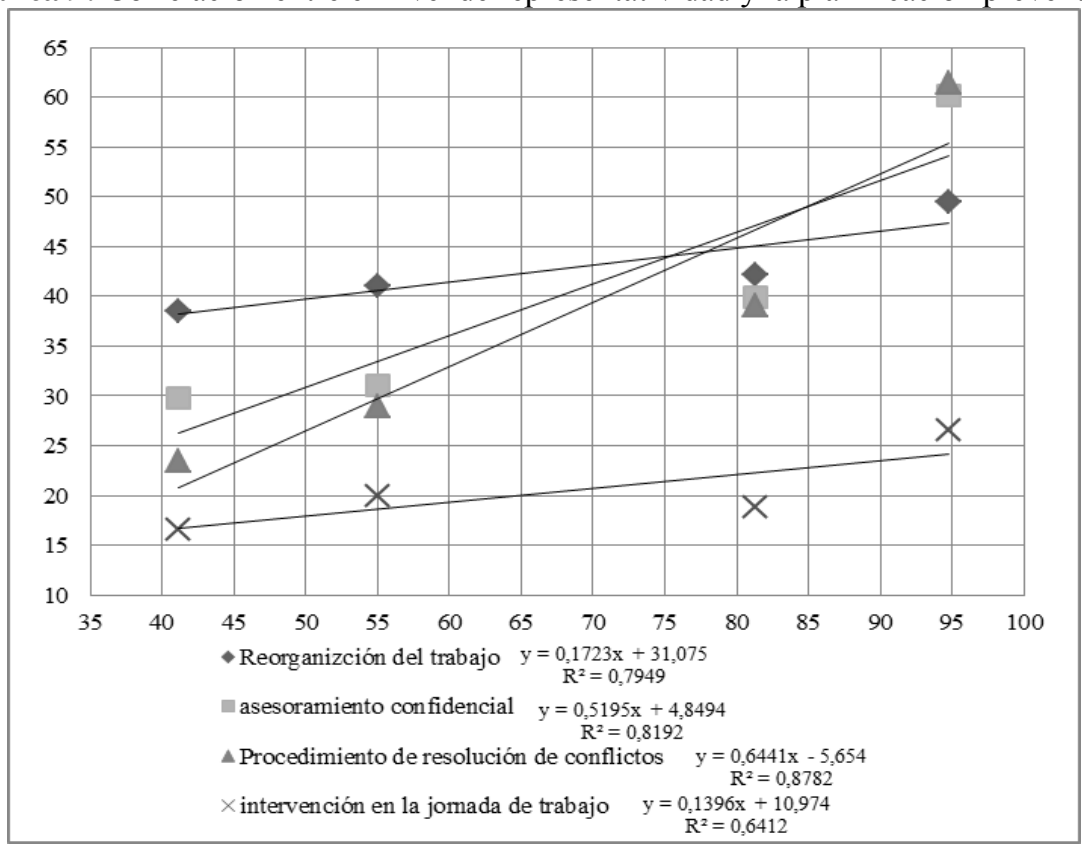

Fuente: Encuesta ESENER-2-España (INSHT, 2014) y ESENER-2 (EU-OSHA, 2014). Elaboración propia

Del mismo modo que ocurría en la fase de evaluación de riesgos psicosociales, la presencia de los representantes de los trabajadores en los centros de trabajo mantiene una fuerte correlación positiva con el establecimiento de medidas de prevención de tales riesgos $(\mathrm{r}=0,89 ; \mathrm{p}<0,04 ; \mathrm{r}=0,91 ; \mathrm{p}<0,01 ; \mathrm{r}=0,93 ; \mathrm{p}<0,005 ; \mathrm{r}=0,81 ; \mathrm{p}<0,01)$, siendo el factor determinante explicativo de la existencia del $79,4 \%$ de las medidas de prevención establecidas para reorganizar el trabajo, del 81,9\% para el asesoramiento confidencial, del $87,8 \%$ para la resolución de conflictos y del $64,1 \%$ de intervenciones sobre la jornada laboral. No obstante, se observa como la evolución de la tasa de planificación de medidas no es tan marcada como en el caso de la evaluación de riesgos, y su nivel de implementación es reducido puesto que solo en las empresas de más de 250 se alcanzan algunos valores cercanos al $60 \%$.

En términos comparativos (gráfica 10), los sistemas de relaciones laborales nórdico y centroeuropeo presentan niveles de implementación de las medidas preventivas superiores a los del área latina como resultado, fundamentalmente, de la más amplia cobertura de sus modelos de representación de los trabajadores, tanto genérico como específico, como hemos tenido ocasión de comprobar supra (vid, gráficas 2 y 6 ).

En referencia, a la última fase del proceso preventivo (gráfica 11), el impacto de la representación de los trabajadores presente también una fuerte correlación positiva con el establecimiento de planes, programas y procedimientos $(r=0,8916 ; p<0,04$. para programas 
frente al estrés; $r=0,9051 ; \mathrm{p}<0,01$ y $\mathrm{r}=0,937 ; \mathrm{p}<0,005$ para planes y procedimientos de acción frente amenazas o hostigamiento intra y extra empresarial respectivamente).

Gráfica 10: Empresas con planes de prevención, según sistemas de RR.LL.

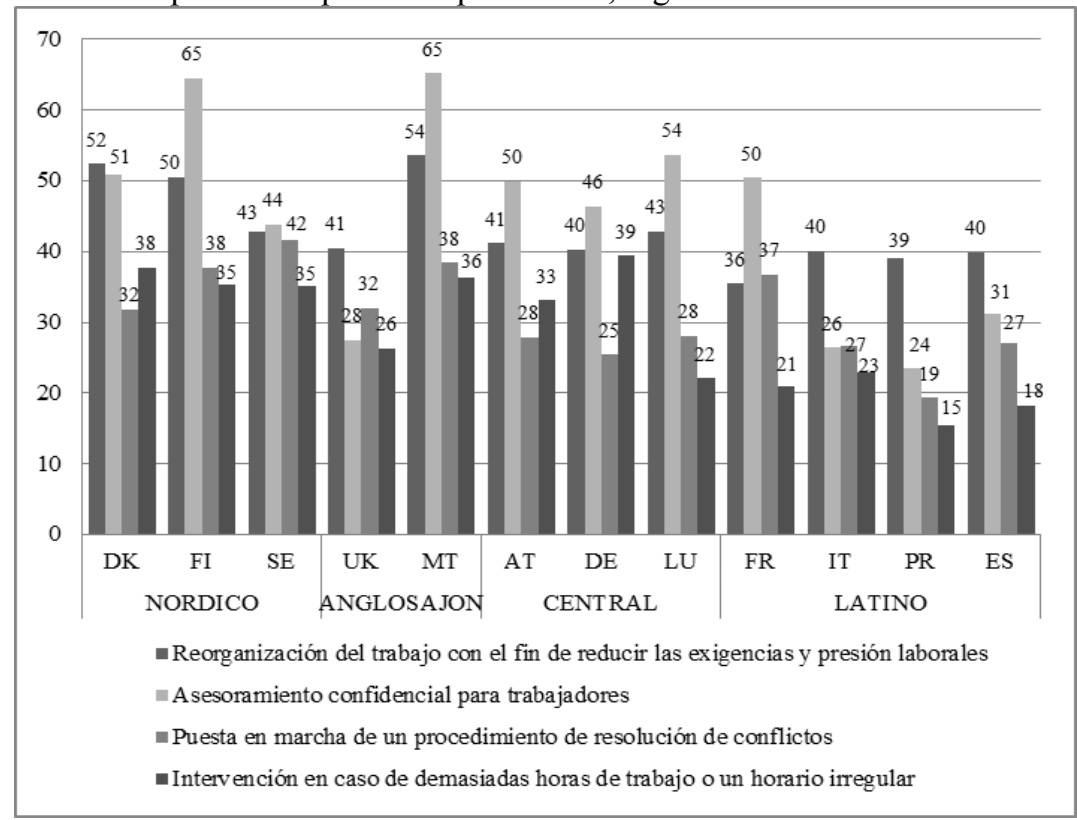

Fuente: Encuesta ESENER-2 (EU-OSHA, 2014). Elaboración propia

Gráfica 11: Correlación entre el nivel de representatividad y la implementación de planes de prevención

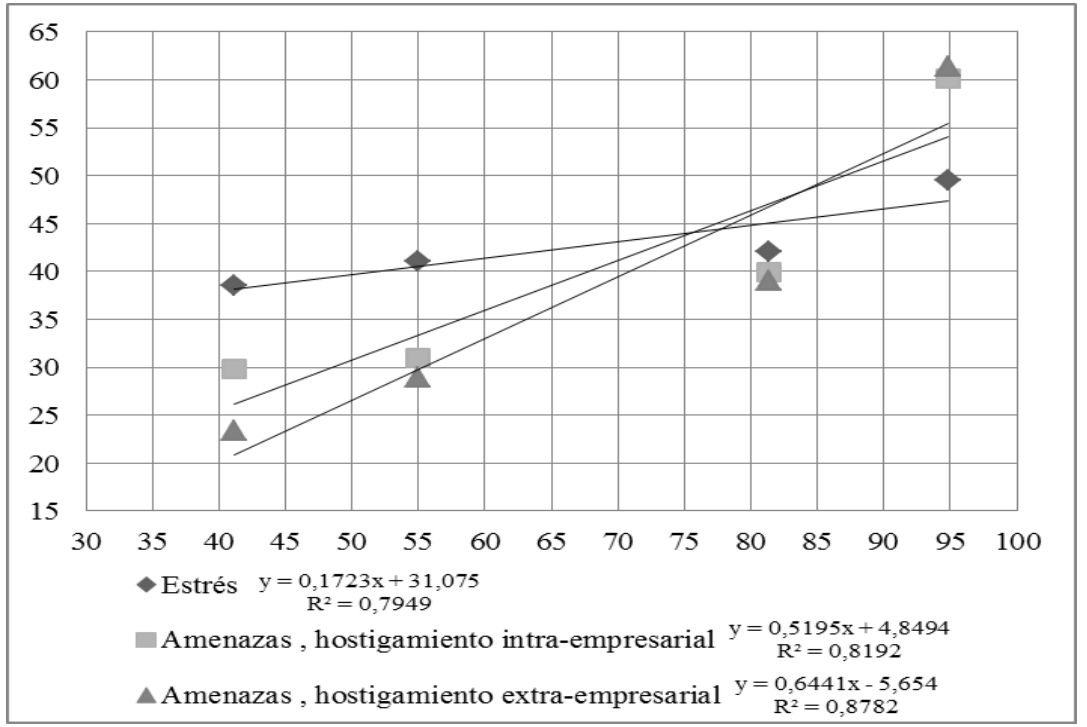

Fuente: Encuestas ESENER-2-España (INSHT, 2014) y ESENER-2 (EU-OSHA, 2014). Elaboración propia 
Lo dicho pone de manifiesto que una vez superado el riesgo se hace visible el problema y los propios trabajadores son los que comunican y denuncian dichas situaciones, exigiendo de la empresa la adopción de las necesarias medidas preventivas.

\section{CONCLUSIONES}

Consideramos, finalmente, que la evidencia empírica aportada en el curso de nuestra investigación, permite confirmar la hipótesis inicial relativa a la existencia de una relación positiva entre la participación de los trabajadores, a través de sus sistemas de representación en la empresa, y los índices de salud laboral, derivada de su impacto sobre las diferentes fases del proceso de prevención de riesgos.

La presencia e intervención de los representantes de los trabajadores, de carácter tanto general (sindical) como específico (funcional) resulta siempre relevante, manteniendo un alto grado de correlación positiva con las diferentes fases del ciclo preventivo: desde la identificación de factores de riesgo al establecimiento, implementación y evaluación de planes de prevención, pasando por el diseño de actividades preventivas, de formación, etc.

Con todo, el efecto combinado de la recesión económica y la desregulación contractual derivada de la reforma laboral, han provocado una importante reducción de la cobertura y eficacia del sistema de representación, lo que estaría debilitando dicha correlación, con el resultado de un repunte sostenido en los índices de siniestralidad.

Parece razonable, en consecuencia, apelar a la recuperación del diálogo social para la implementación de medidas orientadas a mejorar la cantidad y calidad del empleo, reduciendo las altas tasas de temporalidad, así como la cobertura y funcionalidad de los sistemas de representación y participación en materia de prevención de riesgos laborales explorando, a través de la negociación colectiva, nuevas formas de intervención que, como los delegados territoriales de prevención, se han demostrado eficaces en otros países, en defensa de la calidad de trabajo y la salud de los trabajadores y trabajadoras.

\section{BIBLIOGRAFÍA}

Alós, R.; Beneyto, P. y Jódar, P. (2015) La representación sindical en España. Madrid: Fundación $1^{\circ}$ de Mayo

Alós, R.; Beneyto, P. y Jódar, P. (2017) "Reforma laboral y desregulación del mercado de trabajo". Anuario IET de Trabajo y Relaciones Laborales, 4: 73-86, DOI: https://doi.org/10.5565/rev/aiet.55

Benavides, F.; López-Ruiz, M. y Castejón, E. (2008) “Tendencia secular de las lesiones por accidentes de trabajo en España, 1904-2005”. Archivos de Prevención de Riesgos Laborales, 11(3): 141-147.

Beneyto, P. (2014): La representación de los trabajadores en la Unión Europea. Madrid: Fundación $1^{\circ}$ de Mayo, en: http://www.derecho.uba.ar/institucional/2014-representacion-de-los-trabajadores-enespana-y-ue.docx [consulta 10/04/2018].

Boix, P. y Vogel, L. (2006) "Participación de los trabajadores". En C. Ruiz- Frutos, J. Delclòs, A.M. García y F. Benavides (Coords.) Salud laboral. Conceptos y técnicas para la prevención de riesgos laborales. Barcelona: Elsevier, pp. 165-178.

Castejón, E., y Crespán, X. (2007) "Accidentes de trabajo: [casi] todos los porqués", Cuadernos de Relaciones Laborales, 25(1):13-57.

Directiva del Consejo 89/391/CEE. Directiva del Consejo relativa a la aplicación de medidas para promover la mejora de la seguridad y de la salud de los trabajadores en el trabajo Boletín Oficial del Estado, nº183, 1989, 29 de junio, accesible en: https://www.boe.es/buscar/doc.php?id=DOUE-L1989-80648 [consulta 14/04/2018]. 
European Foundation for the Improvement of Living and Working Conditions (Eurofound) (2015) Sixth European Working Conditions Survey, en: https://www.eurofound.europa.eu/surveys/europeanworking-conditions-surveys/sixth-european-working-conditions-survey-2015 [consulta 18/04/2018].

European Statistical Office (Eurostat) (2018) Accidents at work by sex, age and NACE Rev. 2 activity (A, $C-N)$, en: http://appsso.eurostat.ec.europa.eu/nui/show.do?dataset=hsw_mi01\&lang=en [consulta 10/04/2018].

European Agency for Safety and Health at Work (EU-OSHA) (2014): Second European Survey of Enterprise son New and Emerging risks (ESENER-2), en: https:/osha.europa.eu /es/tools-andpublications/second-european-survey-enterprises-new-and-emerging-risks-esener/view [consulta 16/04/2018].

European Agency for Safety and Health at Work (EU-OSHA) (2017) Worker participation in the management of occupational safety and health: qualitative evidence from ESENER-2, en https://osha.europa.eu/es/tools-and-publications/publications/worker-participation-managementoccupational-safety-health/view [consulta 22/04/2018].

Fulton, L. (2013) Health and Safety Representation in Europe. Brussels: European Trade Union Institute (ETUI), en: https://www.worker-participation.eu/National-Industrial-Relations/AcrossEurope/Health-and-Safety2 [consulta 16/04/2018].

García, M.; Cárcoba A.; Menéndez A.; Morón R. y Vogel L. (2015) Qué hacemos con el deterioro de la salud laboral y cómo avanzar en la igualdad y los derechos de los trabajadores en tiempos de crisis. Madrid: Akal.

Instituto Nacional de Estadística (INE) (2018) Encuesta de Población Activa (EPA), en: https://www.ine.es/dyngs/INEbase/es/operacion.htm?c=Estadistica_C\&cid=1254736176918\&menu $=$ resultados\&idp=1254735976595 [consulta 13/04/2018].

Instituto Nacional de Seguridad e Higiene en el Trabajo (INSHT) (2009) Informe de la Encuesta Nacional de Gestión de la Seguridad y Salud en las Empresas (ENGE 2009). Madrid: Instituto Nacional de Seguridad e Higiene en el Trabajo, en: http:/www.oect.es/Observatorio/Contenidos/ InformesPropios/Desarrollados/Ficheros/Informe_\%20ENGE\%202009.pdf [consulta 18/04/2018].

Instituto Nacional de Seguridad e Higiene en el Trabajo (INSHT) (2014) Encuesta Nacional de Gestión de Riesgos Laborales en las Empresas. ESENER-2-España Madrid: Instituto Nacional de Seguridad e Higiene en el Trabajo, en: http://www.oect.es/InshtWeb/Contenidos/Documentacion/FICHAS \%20DE\%20PUBLICACIONES/EN\%20CATALOGO/OBSERVATORIO/ESENER\%20dos.pdf [consulta 4/05/2018].

Instituto Nacional de Seguridad e Higiene en el Trabajo (INSHT) (2017): Encuesta Nacional de Condiciones de Trabajo. $6^{a}$ EWCS-España. Madrid: Instituto Nacional de Seguridad e Higiene en el Trabajo, en: http://www.insht.es/InshtWeb/Contenidos/Documentacion/ENCT\%202015.pdf [consulta 7/05/2018].

Lewchuk, W.; Wolff, A.; King, A. y Polanyi, M. (2003) "From job strain to employment strain: Health effects of precarious employment". Just Labour, 3: 23-35.

Ley 31/1995. Ley de Prevención de Riesgos Laborales (LPRL) Boletín Oficial del Estado, n²69, 1995, 8 de noviembre, accesible en: https://www.boe.es/buscar/act.php?id=BOE-A-1995-24292 [consulta $14 / 04 / 2018]$.

Ley 9/1987. Ley de Órganos de Representación, Determinación de las Condiciones de Trabajo y Participación del Personal al Servicio de las Administraciones Públicas Boletín Oficial del Estado, $\mathrm{n}^{\circ} 144,1987,17$ de junio, accesible en: https://www.boe.es/buscar/doc.php?id=BOE-A-1987-14115 [consulta 16/04/2018].

Linares, P. y López, V. (2016) La participación de los trabajadores y las trabajadoras en salud laboral en España. Red Eurolatinoamericana de Análisis de Trabajo y Sindicalismo (RELATS), en: www.relats.org/documentos/SST.LinaresLopez2016.pdf [consulta 8/04/ 2018].

Ministerio de Empleo y Seguridad Social (2016) Informe anual de la Inspección de trabajo y la Seguridad Social 2016. Dirección General de la Inspección de Trabajo y Seguridad Social, en: http://www.empleo.gob.es/itss/ITSS/ITSS_Descargas/Que_hacemos/Memorias/Memoria_2016.pdf [consulta 22/09/2018].

Ministerio de Trabajo (2004-2017) Anuario de Estadísticas Laborales. Condiciones de Trabajo y 
Relaciones Laborales. Accidentes de trabajo, en: http://www.mitramiss.gob.es/es/estadisticas/ anuarios/2017/index.htm [Consulta 14/04/2018].

Ministerio de Trabajo (2017) Anuario de Estadísticas Laborales. Mercado de trabajo. Afiliación de Trabajadores al Sistema de la Seguridad Social, en http://www.mitramiss.gob.es/es/estadisticas/ anuarios/2017/index.htm [Consulta 14/04/ 2018].

Ministerio de Trabajo (2007-2014) Boletín de Estadísticas Laborales, en http://www.mitramiss.gob.es/ estadisticas/bel/welcome.htm [consulta 13/04/2018].

Ministerio de Trabajo (2007-2017) Estadística de Convenios Colectivos de Trabajo (ECCT), en: http://www.mitramiss.gob.es/estadisticas/cct/welcome.htm [consulta 14/04/2018].

Ministerio de Trabajo (2010) Encuesta de Calidad de Vida en el Trabajo (ECVT), en: http://www.empleo.gob.es/estadisticas/ecvt/welcome.htm_[consulta 22/04/2018].

Narocki, C.; López Jacob, M.J.; Canaleta, E.; Morán, P. y Briso-Montiano, P. (2011) Impacto del asesoramiento sindical en salud laboral. Madrid: Instituto Sindical de Trabajo, Ambiente y Salud, en: $\quad$ http://www.istas.ccoo.es/descargas/Informe\%20impacto\%20asesoramiento\%202011.pdf [consulta 18/04/2018].

Payá, R. (2014) La participación de los trabajadores en seguridad y salud laboral. Una perspectiva Europea. Madrid: Fundación $1^{\circ}$ de Mayo, en http://www.1mayo.ccoo.es/4b23c27b1099b52a23 a08e6a84d2fdfe000001.pdf [consulta 16/04/2018].

Santos, A.; Gabaldón, D.; Ingellis, G. y Muñoz-Rodríguez, D. (2017) Country report Spain: Worker participation in the management of occupational safety and health. Qualitative evidence from ESENER-2. Luxemburgo: European Agency for Safety and Health at Work (EU-OSHA), en: https://www.aepsal.com/wp-content/uploads/2017/04/ESENER_2_Spain_country_report.pdf [consulta 20/04/2018].

Terrés, F.; Rodríguez, P.; Álvarez, E. y Castejón E (2004) "Economic fluctuations affecting occupational safety. The Spanish case". Occupational Ergonomics, 4 (4): 211-228.

Walters, D. y Nichols, T. (2007) Worker representation and workplace health and safety. New York: Palgrave McMillan.

\section{Breve currículo:}

\section{Raúl Payá Castiblanque}

Graduado en Relaciones Laborales, Master en Dirección y Gestión de Recursos Humanos por la Universidad de Valencia y Técnico en Prevención de Riesgos Laborales, con diez años de experiencia profesional. Investigador FPU en el Departamento de Sociología de la Universidad de Valencia, donde prepara su Tesis doctoral sobre los sistemas europeos de participación de los trabajadores en prevención de riesgos.

\section{Pere J. Beneyto Calatayud}

Doctor en Sociología, profesor titular de la Universidad de Valencia y del programa de post-grado de la UNED. Sus líneas de investigación preferente son el mercado de trabajo, las relaciones laborales y los grupos de interés (sindicatos y asociaciones empresariales), la cualificación y formación de recursos humanos; en torno a las que ha desarrollado su actividad académica y profesional. 